\section{Ocular Toxoplasmosis}

An 8-year-old girl presented with headache and eye strain for one week. History and clinical examination were normal. Ophthalmic examination showed anisometropia, normal intra-ocular pressure and fundus showed focal healed scars of central chorioretinitis close to the macula in right eye (Fig. 1). Toxoplasma serology ( $\operatorname{IgG}$ ) was positive for patient and her mother. Unilateral chorioretinitis can occur in both congenital and acquired toxoplasmosis but presence of anisometropia makes vertical transmission more likely. Ocular involvement in the form of focal necrotizing chorioretinitis and central nervous system lesions develop by adolescence in untreated patients. Other ocular manifestations include optic nerve involvement, vitritis, anterior uvitis and retinal detachment. Majority will have severe visual impairment. Characteristic retinal lesions and positive serology helps in diagnosis. Recurrent or progressive disease can occur because the encysted form of organisms persists throughout life. Suppressive treatment



FIG.1 Scars of healed central chorioretinitis (arrows).

with pyrimethamine, sulfadiazine and folinic acid prevents frequent recurrences of lesions.

RP KARAMBELKAR AND GR KARAMBELKAR Near Bank of India, BP Road, Dehuroad, Pune 412 101, India. rjkarambelkar@yahoo.co.in

\section{Descemet Membrane Breaks Following Forceps Delivery}

A term male neonate born by forceps vaginal delivery presented on day 10 of life with watering and opacification of cornea of left eye. There was associated lacrimation, photophobia and blepharospasm. Eye examination under anaesthesia revealed corneal edema (Fig.1) with normal intraocular pressure. Corneal edema resolved following topical corticosteroids but on repeat examination vertically oriented breaks were noted in descemet membrane (Fig.2b, white double arrow). These breaks were associated with myopic astigmatism of $3.50 \mathrm{D}$ along the axis of break. Refractive correction was prescribed along with standard occlusion therapy. At follow up of 2 months and later at 2 years, refractive error was stable with the best corrected visual acuity of 20/30 in the affected eye.

Forceps injury to eye usually occurs as a result of compression of the globe between the orbital roof and the blade of the obstetric forceps. It is associated with descemet membrane breaks and can cause refractive errors. These breaks being small and obscured by corneal edema are easily missed. A normal intraocular pressure facilitates differentiation from congenital glaucoma. The corneal edema improves within a few weeks, at which time residual
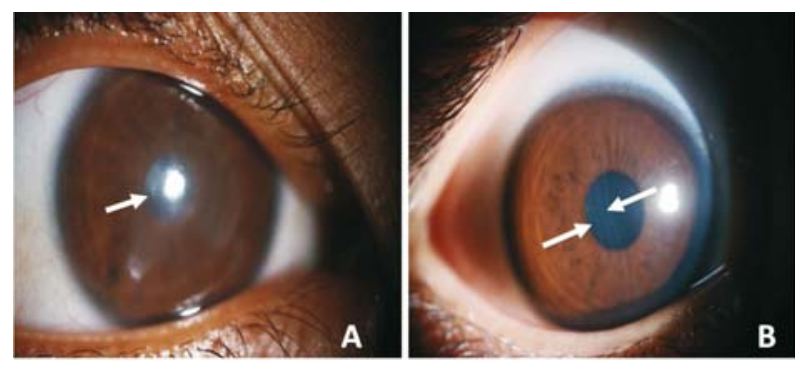

Fig.1 Slit lamp photographs of affected (left) eye; Panel A: on 10 th day of life revealing diffuse corneal edema (white arrow); Panel B: On day 28 of life revealing vertical break at the level of descemet's membrane (white, double arrow).

single or multiple descemet membrane breaks appear as vertical or oblique median striae. These corneal lesions are primarily unilateral and may cause amblyopia. Loss of vision may be because of the opacities of the striae themselves or because of induced astigmatism, which results in amblyopia. Although penetrating keratoplasty has been the treatment of choice, but this leads to frequent complications in children.

Suma Ganesh, Priyanka Arora And *Kamaldeep Arora Department of Pediatric Ophthalmology and Strabismus, Dr Shroff's Charity Eye Hospital; and Department of Pediatrics, All India Institute of Medical Sciences, New Delhi, India. priyankagalhotra@gmail.com 